\begin{tabular}{|l|l|}
\hline $\begin{array}{l}\text { Postprint } \\
\text { Version }\end{array}$ & 1.0 \\
\hline Journal website & http://dx.doi.org/10.1111/jan.12376 \\
\hline Pubmed link & http://www.ncbi.nlm.nih.gov/pubmed/24588648 \\
\hline DOI & $10.1111 /$ jan.12376 \\
\hline
\end{tabular}

This is a NIVEL certified Post Print, more info at http://www.nivel.eu

\title{
Effects of video-feedback on the communication, clinical competence and motivational interviewing skills of practice nurses: a pre-test posttest control group study
}

\author{
JANNEKE NOORDMAN PHD RESEARCHER ${ }^{1, *}$, TRUDY VAN DER WEIJDEN MD PHD PROFESSOR \\ IMPLEMENTATION OF CLINICAL PRACTICE GUIDELINE ${ }^{2}$, SANDRA VAN DULMEN PHD \\ RESEARCH COORDINATOR NIVEL \& PROFESSOR COMMUNICATION IN HEALTH CARE ${ }^{1,3,4}$
}

\section{Abstract}

\section{Aims:}

To examine the effects of individual video-feedback on the generic communication skills, clinical competence (i.e. adherence to practice guidelines) and motivational interviewing skills of experienced practice nurses working in primary care.

\section{Background:}

Continuing professional education may be necessary to refresh and reflect on the communication and motivational interviewing skills of experienced primary care practice nurses. A video-feedback method was designed to improve these skills.

\section{Design:}

Pre-test/posttest control group design.

\section{Methods:}

Seventeen Dutch practice nurses and 325 patients participated between June 2010 June 2011. Nurse-patient consultations were videotaped at two moments (T0 and T1), with an interval of 3-6 months. The videotaped consultations were rated using two protocols: the Maastrichtse Anamnese en Advies Scorelijst met globale items (MAAS-global) and the Behaviour Change Counselling Index. Before the recordings, nurses were allocated to a control or video-feedback group. Nurses allocated to the video-feedback group received video-feedback between T0 and T1. Data were analysed using multilevel linear or logistic regression.

\section{Results:}

Nurses who received video-feedback appeared to pay significantly more attention to patients' request for help, their physical examination and gave significantly more understandable information. With respect to motivational interviewing, nurses who received video-feedback appeared to pay more attention to 'agenda setting and permission seeking' during their consultations. 
Noordman, J., Weijden, T. van der, Dulmen, S. van. Effects of video-feedback on the communication, clinical competence and motivational interviewing skills of practice nurses: pre-test posttest control group study. Journal of Advanced Nursing: 2014, 70(10), 2272-2283

\section{Conclusion:}

Video-feedback is a potentially effective method to improve practice nurses' generic communication skills. Although a single video-feedback session does not seem sufficient to increase all motivational interviewing skills, significant improvement in some specific skills was found. Nurses' clinical competences were not altered after feedback due to already high standards.

\section{[Box 1]}

\section{INTRODUCTION}

Globally, primary care practice nurses monitor the chronically ill and patients at risk for chronic illnesses (e.g. obese patients, who are at risk for developing diabetes type 2 or heart and vascular disease) and provide them with education and support for behaviour change (Katon et al. 2001, Voogdt-Pruis 2011). Practice nurses have to follow clinical guidelines in monitoring and supporting these patients (e.g. guidelines for diabetes type 2, asthma/COPD, heart and vascular disease, quit smoking guideline) (Heiligers et al. 2012).

The level of professional autonomy of primary care nurses varies both within and across countries, but is generally high (Richardson \& Cunliffe 2003, Fairman et al. 2011). In the Netherlands, practice nurses work under supervision of general practitioners, which means that practice nurses cannot diagnose, refer patients or prescribe medicines without permission of a general practitioner. All practice nurses manage consultations independently in a separate consulting room in their general practice (Heiligers et al. 2012). This level of autonomy also applies, for example, to primary care nurses in several states of the USA (Fairman et al. 2011).

In the Netherlands, practice nurses follow a specific education or specialization of 12 years, depending on the educational background (i.e. having a bachelor degree or lower vocational training) (Heiligers et al. 2012). Currently, Dutch practice nurses are taught generic communication skills (e.g. exploring, providing information and advice) and motivational interviewing or 'behaviour change counselling' skills as part of their standard education (Heiligers et al. 2012). However, recent (international) studies among nurses in diabetes management and public health indicate that implementing motivational interviewing in daily care is not so easy (Heinrich et al. 2010, Pfister-Minogue \& Salveson 2010, van Eijk-Hustings et al. 2011, Noordman et al. 2012a). Previous studies suggest that time pressure and the dominance of biomedical aspects during consultations are barriers for implementing motivational interviewing (Heinrich et al. 2010, Voogdt-Pruis 2011). This study was designed to improve the communication and motivational interviewing skills of experienced practice nurses, using video-feedback.

\section{BACKGROUND}

Communication is a nurses’ primary tool during consultations (Rice \& Stead 2009, Noordman et al. 2012b). A promising communication strategy in this respect is motivational interviewing. Studies indicate that motivational interviewing is effective in several domains of behaviour change (Rubak et al. 2005, Martins \& McNeil 2009). Motivational interviewing is a patient-centred approach that focuses on increasing intrinsic motivation to behaviour change by helping patients to explore and to resolve ambivalence between desired behaviour and actual behaviour (Miller 
Noordman, J., Weijden, T. van der, Dulmen, S. van. Effects of video-feedback on the communication, clinical competence and motivational interviewing skills of practice nurses:
pre-test posttest control group study. Journal of Advanced Nursing: 2014, 70(10), 2272-2283

\& Rollnick 2002, Martins \& McNeil 2009). Motivational interviewing skills are supposed to be embedded in an interaction built on more generic communication skills (e.g. providing information, exploring wishes or expectations) and clinical competence (i.e. following practice guidelines) (Kurtz et al. 2005).

Continuing professional education may be necessary to refresh and reflect on the communication and motivational interviewing skills of experienced practice nurses in routine practice. Furthermore, it holds the promise of patients benefitting as a consequence (Davey \& Robinson 2002). Video-feedback, based on video-recorded real-life patient encounters, seems a promising method for continuing professional education (Ivers et al. 2012). A recent meta-analysis shows that video-feedback has a significant positive effect on the key communication skills of several different professionals (e.g. nurses, physicians) (Fukkink et al. 2011). Video-feedback has enhanced physicians' evaluation of their own communication and its impact is more enduring than traditional didactic methods (Coleman 2000, Roter et al. 2004, Ozcakar et al. 2009). A more time-efficient method is the online demonstration of video-recorded communication skills (Kemper et al. 2008). In this context, we designed a video-feedback method, which was found to be acceptable and useful for primary care physicians (Noordman et al. 2011). It is, however, unknown to what extent this method affects the communication skills and motivational interviewing skills of practice nurses.

\section{THE STUDY}

\section{Aim}

The aim of the study was to examine the impact of individual video-feedback on the generic communication skills, clinical competence (i.e. adherence to practice guidelines) and motivational interviewing skills of experienced practice nurses working in primary care.

It is hypothesized that video-feedback improves practice nurses' generic communication skills and motivational interviewing skills. Possibly, nurses' clinical competence (adherence to practice guidelines) will not improve much, because of nurses' accountability (to general practitioners and as stipulated in the contract with the insurer) regarding the compliance with guidelines (Heiligers et al. 2012), as well as their own professional registration requirements and, therefore, expected high standards at baseline.

\section{Design}

Pre-test/posttest control group design (Figure 1).

\section{[FIGURE 1]}

\section{Participants and data collection}

Practice nurses from seven practices were approached by contacting their general practitioner who participated (except for one practice) in an earlier study performed by the Netherlands Institute for Health Services Research (NIVEL) (Noordman et al. 2010) (47\% of the general practitioners responded; six practices did not employ a practice nurse). In addition, general practitioners from one other practice (healthcare centre) contacted us for participation of all of their practice nurses $(n=9)$; all nurses volunteered to take part. The study took place between June 2010-June 2011. Our 
aim was to include 20 practice nurses. Altogether, 20 practice nurses from eight practices in the Netherlands agreed to have series of consecutive consultations videotaped at two moments (T0 and T1), with 3-6 months in between the recording moments. Approximately ten consultations per nurse were recorded on video at T0 and ten consultations per nurse at T1. Patients were approached by a researcher in the waiting room and signed a written informed consent form. Adult patients scheduled for an appointment with the practice nurse were eligible for inclusion (90\% agreed to participate, no dropout). Nurses also signed an informed consent form before the recording of the consultations (Figure 1). All nurses were trained in motivational interviewing (or behaviour change counselling) as part of their education, of which 13 nurses had had more elaborate posteducation training in motivational interviewing prior to our study (Noordman et al. 2012a, 2013).

\section{Allocation to control or video-feedback group}

A pre-test/posttest control group design was used, where all practice nurses were offered feedback (during or after the study); see section 'Web-enabled videofeedback'. Before the recording of the consultations, nurses were allocated to a control or video-feedback group. Nurses allocated to the video-feedback group received video-feedback between T0 (pre-measurement; first video-recording moment) and T1 (postmeasurement; second recording moment), T1 taking place between 1-2 months after the video-feedback. Nurses in the control group received video-feedback after the study. To prevent contamination (i.e. cross-over of information between conditions), participants in the video-feedback group were explicitly asked not to discuss the feedback until all consultations of the control group participants were recorded.

Of the nine nurses from one healthcare centre, five nurses were allocated to the video-feedback group and four to the control group. The other 11 nurses from seven practices were consecutively allocated to the video-feedback or control group per practice.

In the end, ten practice nurses were part of the video-feedback group and seven were part of the control group. Two nurses, assigned to the control group, only participated at T0 (because of having become unemployed or being too busy at T1) and were left out of the analysis. Also, one other nurse (assigned to the control group) was in the middle of her education between $\mathrm{T} 0$ and $\mathrm{T} 1$ and was left out of the analysis, because the education in between could influence our outcomes (i.e. to avoid co-interventions).

\section{Ethical considerations}

The study was carried out according to Dutch privacy legislation and adhered to the Dutch Data Protection Authority accepted privacy regulations. Prior to the consultation, all nurses and patients signed an informed consent form. According to Dutch legislation, approval by a medical ethics committee was not required for this observational study.

\section{Observations}

Two observers (i.e. trained researchers with a social science background) independently coded the videotaped consultations, by means of two validated protocols: (1) the Maastrichtse Anamnese en Advies Scorelijst met globale items (MAAS-global) (van Thiel et al. 2000); and (2) the Behaviour Change Counselling Index (BECCI) (Lane 2002, Lane et al. 2005). These protocols were programmed 
and rated in Observer software (Noldus et al. 2000). To assess nurses’ generic communication skills and clinical competence, the MAAS-global was used. The MAAS-global comprises of three sections: communication skills for each separate consultation phase (from introduction to evaluation of consultation), general communication skills (e.g. exploration, empathy) and clinical aspects (adherence to practice guidelines). Each item is assessed on a scale ranging from 0 'not present'-6 'excellent'. Some items (e.g. follow-up consultation in case of a first encounter) could be scored as 'not applicable' and were left out of the analyses.

To code nurses' application of motivational interviewing skills, the BECCI was used. This protocol covers 11 items, subdivided into four domains: agenda setting and permission seeking (two items, Cronbach's $\alpha=0.49$ ); the why and how of change in behaviour (five items, $\alpha=0.71$ ); the whole consultation (three items, $\alpha=0.76$ ); and talk about targets (one item). Items are scored on a five-point Likert-scale ranging from 'not at all' to 'a great extent'. As recommended by the author of this protocol (Lane 2002), not applicable items were replaced by mean substitution. Both observation protocols are added as supplementary web-based materials (See JAN website).

\section{Validity and Reliability}

To establish interrater reliability, ten per cent of the 325 consultations were coded by both observers. Interrater agreement between observers was sufficiently high, with average Kappa scores (Sim \& Wright 2005) of 0.85 (range 0.72-1·00) for the MAAS-global and $0 \cdot 85$ (range 0.77-0.95) for the BECCI.

\section{Web-enabled video-feedback}

All practice nurses received individual 'web-enabled video-feedback' during (videofeedback group) or after (control group) the study. Previously, this method was used to assess the communication skills of experienced primary care physicians (Noordman et al. 2011). For the present purpose, two consultations were selected for each nurse: one consultation to show their good points and one consultation to demonstrate their improvement regarding their communication behaviour. The two consultations were selected based on the mean scores of their communication in the other recorded consultations (i.e. scores on the MAAS-global and BECCI).

Nurses received an email containing two secured individual web links on which they could observe their two selected video-recorded consultations. To watch the selected consultations, nurses clicked on the web links. They could watch the consultations, during 1-2 weeks, at any time or place (e.g. at home or the office). After watching, nurses received feedback, by one of two researchers, on their generic communication skills, clinical competence (i.e. adherence to guidelines) and motivational interviewing skills, during an individual face-to-face 1-hour meeting with a researcher. The feedback was structured around an individual feedback report. The feedback report described the communication behaviour of the nurse (i.e. assessment of the MAAS-global and BECCI) in comparison with the communication behaviour of the other participating nurses and concluded with a 'take-home message' where the good elements and improvement points of their communication skills were summarized. The aim of the feedback session was to make nurses aware of the strengths and weaknesses in their communication behaviour and to give direction in applying a particular communication skill during a specific consultation. Before 
Noordman, J., Weijden, T. van der, Dulmen, S. van. Effects of video-feedback on the communication, clinical competence and motivational interviewing skills of practice nurses:
pre-test posttest control group study. Journal of Advanced Nursing: 2014, 70(10), 2272-2283

providing feedback, the two researchers discussed their findings and method of delivery with each other.

At the start of the feedback session, nurses were asked to evaluate their own communication behaviour (in general and during the selected consultations in particular). No directions were given on evaluating their own communication behaviour. At the end of the feedback session, nurses were asked to reflect on the usefulness and efficiency of the feedback (method) (Noordman et al. 2011).

\section{Data analyses}

First, characteristics of the practice nurses and patients in the control and videofeedback group were described. Differences between the groups were tested using an independent t-test for continuous variables and chi-square test for dichotomous and categorical variables. Second, the average scores for the BECCI domains and items of the MAAS-global were described for the control and video-feedback group on T0 (pre-measurement).

Third, multilevel linear and logistic regression analyses were performed. Three levels were used: patients (level 1), time of measurement (level 2) and practice nurses (level 3). 'Time of measurement' was used as level 2 because of different patients at T0 and T1 respectively. First, a null model for every dependent variable (e.g. BECCI domain or MAAS-global item) was created, thereby correcting for patient's social demographic characteristics - age, gender and ethnicity (model 0). We created these separate models for every dependent variable to avoid multiple testing. Next, we added time of measurement (T0 vs. T1), research group (control vs. video-feedback group) and the interaction term between time of measurement and research group to the model (model 1). Multilevel linear regression was used to determine the association between the four domain scores of the BECCI, BECCI total score, the BECCI items (almost all) and MAAS-global items (dependent variables) with the interaction term between time of measurement and research group (e.g. the effect of video-feedback) (model 1). The MAAS-global items 'Introduction', 'Request for help', 'Evaluation of consultation' and 'Emotions' were coded as dichotomous variables and analysed with multilevel logistic regression, using the second order marginal quasi-likelihood (MQL) method. The descriptive analyses were performed in Stata 11 (StataCorp LP, College Station, TX, USA) and the multilevel analyses in MLwiN 2.25 (Goldstein et al. 1998).

\section{RESULTS}

\section{Sample}

At pre-measurement (T0), 163 consultations between 17 practice nurses and 163 patients were analysed. At postmeasurement (T1), 162 consultations between 17 practice nurses and 162 patients were analysed (Figure 1).

\section{Characteristics and communication skills of practice nurses and characteristics of patients}

In Table 1, the characteristics of practice nurses, patients and nurses' generic communication, clinical competence and motivational interviewing skills in the video-feedback and control group at pre-measurement (T0) are presented. 
Noordman, J., Weijden, T. van der, Dulmen, S. van. Effects of video-feedback on the communication, clinical competence and motivational interviewing skills of practice nurses: pre-test posttest control group study. Journal of Advanced Nursing: 2014, 70(10), 2272-2283

\section{[TABLE 1]}

At pre-measurement, nurses in the video-feedback group did not differ significantly with respect to gender, working experience and age from nurses in the control group. However, the groups differed in whether they had received posteducational training in motivational interviewing $(\mathrm{P}<0 \cdot 01)$. All nurses $(\mathrm{n}=10)$ in the video-feedback group and three nurses in the control group received posteducational motivational interviewing training prior to T0, i.e. prior to our study. This motivational interviewing training varied between $1 / 2$ day and six half days (Noordman et al. 2012a).

At pre-measurement (T0), nurses in the video-feedback group did not differ from nurses in the control group with respect to their generic communication, clinical competence and motivational interviewing skills. Except for one generic communication skill, nurses in the control group 'structured' their consultations significantly more compared with nurses in the video-feedback group $(\mathrm{P}=0 \cdot 04)$. At pre-measurement, patients in the video-feedback group differed from patients in the control group with respect to age and ethnicity $(\mathrm{P}<0 \cdot 01)$. Therefore, further analyses controlled for patients' characteristics. No significant differences were found between the mean consultation duration (in minutes) and the type of consultation (i.e. first consultation or follow-up consultation) of the video-feedback and control group at pre-measurement.

\section{Effect of video-feedback on practice nurses' generic communication skills} In Table 2, the regression coefficients of practice nurses' generic communication skills (according to the MAAS-global) at pre-measurement vs. postmeasurement are depicted. Comparisons were made between the scores of the video-feedback group and the control group, at postmeasurement vs. pre-measurement. Three significant differences emerged. At postmeasurement, nurses in the video-feedback group appeared to pay more attention to patients' request for help $(\mathrm{P}<0 \cdot 01)$ and to the physical examination (for example, measuring blood pressure) $(\mathrm{P}<0 \cdot 01)$. Furthermore, they gave more understandable information $(\mathrm{P}=0 \cdot 02)$ during their consultations. In addition, a trend appeared for the item 'exploration' $(\mathrm{P}=0 \cdot 07)$. At postmeasurement, nurses in the video-feedback group tend to pay more attention to exploring patients' wishes, expectations and responses. The significant regression coefficients range from $0 \cdot 61-1 \cdot 66$. Hence, differences are small. Intraclass correlations (ICCs) ranged from 0.00 for 'request for help' (no difference between nurses) to $0 \cdot 28$ for 'physical examination' (quite large difference between nurses' application of the skill), pointing to the need to use multilevel analyses.

\section{[TABLE 2]}

\section{Effect of video-feedback on practice nurses' clinical competence}

In Table 3, the regression coefficients of nurses' clinical competence (i.e. adherence to guidelines; according to the MAAS-global) at pre-measurement vs.

postmeasurement are presented. No differences were found between the scores of the control and video-feedback group at postmeasurement. ICCs ranged from $0 \cdot 04$ (for the competence 'management') to $0 \cdot 18$ (for the competence 'physical examination'). 
Noordman, J., Weijden, T. van der, Dulmen, S. van. Effects of video-feedback on the communication, clinical competence and motivational interviewing skills of practice nurses: pre-test posttest control group study. Journal of Advanced Nursing: 2014, 70(10), 2272-2283

[TABLE 3]

\section{Effect of video-feedback on practice nurses' motivational interviewing skills}

Multilevel analyses showed one significant difference on nurses' motivational interviewing skills, measured with the BECCI (Table 4). At postmeasurement, nurses in the video-feedback group appear to pay more attention to 'agenda setting and permission seeking' (domain 1$)(P=0 \cdot 01)$. Analysis of the underlying items of domain 1 (1. 'nurse invites the patient to talk about behaviour change' and 2. 'nurse demonstrates sensitivity to talking about other issues') revealed that both items showed a significant effect in favour of the video-feedback group at postmeasurement, with $P$ values of 0.04 and 0.02 respectively (results not shown in Table 4). No other significant differences were found between the scores of the control and the video-feedback group at postmeasurement. The regression coefficients range from $0 \cdot 01$ to $0 \cdot 39$, indicating small differences. ICCs ranged from $0 \cdot 00$ (for domains 3 and 4) $-0 \cdot 16$ (for domain 2).

\section{[TABLE 4]}

\section{Practice nurses' evaluation of the video-feedback}

All practice nurses were satisfied with the feedback they received. Also, the method of feedback was acknowledged as beneficial. Nurses were particularly satisfied with the option to watch their video-recorded consultations (using the web-link) and the structured feedback report. The face-to-face 1-hour session where feedback was provided was also highly appreciated. Nurses valued the extensiveness of the feedback and measurements used. Furthermore, nurses stated that they recognized themselves in the provided feedback and expected that they were able to 'put the feedback into practice'. However, there were also some less positive comments about the (method of) feedback. Some nurses had to watch their recorded consultations at home, because they were not able to watch these at work (due to security/network restrictions of the practice). Furthermore, one nurse (from the control group) did not want to watch her recorded consultations, because she thought it would be too confronting. Still, she did receive the face-to-face feedback and written report. In total, 15 of the 17 nurses had watched their consultations before the face-to-face feedback session as intended. In addition, after the feedback session, one nurse suggested to provide feedback in future on two, quite similar consultations instead of the ones we selected. In contrast, her colleague recommended using two different consultations in the future, because she found the two we selected too similar. Finally, one nurse mentioned that the period between the first recording of the consultations (pre-measurement) and the feedback was too long (i.e. $2 \cdot 5$ months). Nevertheless, the video-recorded consultations did prompt her memory.

\section{Discussion}

The overall aim of our research was to evaluate the effects of individual videofeedback on the generic communication skills, clinical competence (i.e. adherence to guidelines) and motivational interviewing skills of experienced practice nurses working in primary care.

Providing individual video-feedback to experienced practice nurses resulted in improvement of some of their generic communication skills (i.e. request for help, physical examination and information giving) and motivational interviewing skills 
(i.e. agenda setting and permission seeking). In addition, all nurses evaluated the video-feedback (method) as useful. In accordance with our previous feedback study (Noordman et al. 2011), nurses were particularly satisfied with the option to watch their video-recorded consultations (using the web-link) and with the structured feedback report.

No effect of the video-feedback was found on nurses' clinical competence, possibly due to their high competence level, already at pre-measurement (ceiling effect). Nurses adhered to practice guidelines to a great extent. Previous research suggests that adhering to the biomedical aspects of guidelines can conflict with a patientcentred approach such as motivational interviewing (Heinrich et al. 2010, Berger 2011, Voogdt-Pruis 2011). More specifically, it is possible that adhering to quality indicators based on practice guidelines during consultations is an impediment to the use of motivational interviewing. Such quality indicators may be part of financial incentive and reimbursement systems and therefore demand practice nurses to meet certain task requirements, but nurses also need to take the patients' motivation into account as part of motivational interviewing. Further research should examine whether these seemingly conflicting tasks can become intertwined or whether combining these tasks in an appropriate way is too much to ask for.

Furthermore, a single session video-feedback may not be sufficient to improve nurses' motivational interviewing skills to a great extent. Still, we did find some significant improvement in nurses' motivational interviewing skills after only one feedback session. This is remarkable, as nurses who were more extensively trained in motivational interviewing (prior to our study) did not differ from nurses who were not trained in motivational interviewing at pre-measurement in our study. However, it is possible that a basic amount of motivational interviewing knowledge is needed to increase the effect of individual feedback. So far, it is unknown how much and which training is actually needed for motivational interviewing (Britt et al. 2004). Video-feedback did positively affect some nurses' generic communication skills and, to a lesser extent, also nurses' exploring skills. Especially, 'exploring' is known to be a difficult skill to apply (Olde Hartman et al. 2013), for which any improvement is welcome.

Future studies are necessary to examine the long-term effects of video-feedback on nurses' communication skills and how much feedback or training is needed to improve the implementation of motivational interviewing in daily practice. Previous research among physicians suggests that the most effective feedback is systematic feedback (i.e. according to a predefined protocol) provided over several years (Veloski et al. 2006), as many practitioners tend to return to old counselling habits after a few months (Miller \& Mount 2001). In this respect, it is important to make sure that the training is correctly implemented in daily practice (to safeguard the 'transfer' from knowledge to practice) and to examine what hinders this process, by means of video-observation research (van Dulmen 2012). In addition, it is also important to investigate the role of patients in facilitating or hindering nurses' application of motivational interviewing. It is possible that motivational interviewing is not suitable for all patients and that some patients even show resistance to motivational interviewing (Heinrich 2011). However, improving nurses’ motivational interviewing skills can improve care for patients with (risk of) longterm illness. Several studies found that motivational interviewing has led to positive psychological, physiological and lifestyle outcomes for patients and is particularly 
Noordman, J., Weijden, T. van der, Dulmen, S. van. Effects of video-feedback on the communication, clinical competence and motivational interviewing skills of practice nurses:
pre-test posttest control group study. Journal of Advanced Nursing: 2014, 70(10), 2272-2283

suitable in the management of long-term illness (Rubak et al. 2005, Knight et al. 2006, Britt \& Blampied 2010). Adapting motivational interviewing to patient's stage of change (i.e. intention to change behaviour) could be even more helpful (Noordman et al. 2013). Furthermore, previous studies demonstrate that the involvement of a practice nurse leads to improved care and access to care for chronically ill patients, especially for patients with diabetes type 2 (Richards et al. 2000, Heiligers et al. 2012).

The findings have implications for nursing practice. First, given the comparable level of professional autonomy of Dutch primary care nurses and nurses in other Western countries like the USA (Fairman et al. 2011) and the presence of primary care nurses in other countries like the UK, Sweden, Finland, Canada, Australia and New-Zealand (Bourgueil et al. 2005), findings of this study are valuable to countries outside the Netherlands. However, as recently pointed out by Gardner et al. (2013), globally the level and roles of nursing are variously defined and lack universal nomenclature. This should be taken into account when transferring our findings to nursing practice in other countries or settings (e.g. hospitals). Secondly, continuing professional education is recognized as beneficial internationally (Tame 2013). As this study shows, video-feedback as method for continuing professional education seems helpful for primary care nurses and should therefore perhaps be provided on a regular basis (for example, every year). Video-feedback could also be provided among peers, e.g. colleagues, on the condition that nurses have a common understanding of the communication approach (i.e. motivational interviewing) and support each other (Towle et al. 2006, van Eijk-Hustings et al. 2011). Finally, integrating motivational interviewing skills with practice standards (van Weel-Baumgarten et al. 2013) seems useful to improve nurses' communication during their consultations.

\section{Strengths}

This is the first study, to our knowledge, that aims to improve the communication and motivational interviewing skills of experienced practice nurses using videofeedback.

A strength of our study is that it is based on the (quantitative) assessment of real-life consultations instead of relying on more subjective methods as, for example, questionnaires. Also, practice nurses evaluated their own communication and the video-feedback (method).

\section{Limitations}

There are also some limitations. Due to pragmatic reasons (i.e. taken into account their possibilities for recording days in relation to their part-time job and consecutively allocated at time of application), stratified allocation and blinding of practice nurses was not possible. In addition, observers were not entirely blinded to the intervention. This could have influenced our outcomes. Furthermore, the number of practice nurses $(n=17)$ was too small to allow for correction of nurses' characteristics in the multilevel analyses (i.e. prior posteducational motivational interviewing training and amount of 'structuring' differed between video-feedback and control group at pre-measurement). However, we did control for patient characteristics. In addition, the second-order MQL method was used for multilevel logistic regression, as first- and second-order predictive quasi-likelihood (PQL) could not be performed due to too many values of zero (for 
example, 'introduction’ was not present many times and scored as zero). MQL could underestimate the random parameters (variance) compared with PQL.

Another limitation was the low Cronbach's alpha score on BECCI domain 1 (two items, $\alpha=0 \cdot 49$ ). Nevertheless, analysis of the underlying two items of domain 1 revealed the same significant association(s). Furthermore, the video-feedback resulted in a short-time improvement of some of nurses' generic communication skills and motivational interviewing skills. Long-term effects have yet to be investigated, including a larger number of practice nurses. Lastly, it was not possible to determine whether or not the sample of practice nurses (PNs) was representative for the Dutch population of PNs, as numbers of PNs working in the Netherlands are incomplete.

\section{CONCLUSION}

This study suggests that video-feedback, including a face-to-face session and written report, is a potentially effective method to improve practice nurses' generic communication skills. In addition, practice nurses evaluated the video-feedback (method) as useful. However, one-time video-feedback may not be sufficient to increase motivational interviewing skills. Still, we did find some significant improvement in nurses' motivational interviewing skills after only one feedback session. Nurses' clinical competence, i.e. their adherence to practice guidelines, was not altered after the feedback, possibly due to already high standards (i.e. ceiling effect).

\section{ACKNOWLEDGEMENTS}

We would like to thank the patients and practice nurses for their participation. We also thank Inge van der Lee and Nienke Franse for their help in data acquisition and coding of the video-recorded consultations. Thanks to Marcus Winckers for creating the web-enabled links to the video-recorded consultations and Peter Spreeuwenberg for his statistical advice.

\section{FUNDING}

This study was funded by the Dutch Ministry of Health, Welfare and Sport. The Ministry had no role in the collection, analysis, interpretation, writing or decision to submit this article.

\section{CONFLICT OF INTEREST}

No conflict of interest has been declared by the authors.

\section{AUTHOR CONTRIBUTIONS}

All authors have agreed on the final version and meet at least one of the following criteria [recommended by the ICMJE (http://www.icmje.org/ethical_1author.html)]:

- substantial contributions to conception and design, acquisition of data, or analysis and interpretation of data;

- drafting the article or revising it critically for important intellectual content. 
Noordman, J., Weijden, T. van der, Dulmen, S. van. Effects of video-feedback on the communication, clinical competence and motivational interviewing skills of practice nurses: a pre-test posttest control group study. Journal of Advanced Nursing: 2014, 70(10), 2272-2283

\section{REFERENCES}

Berger M.Y. (2011) De Patiënt, z'n Klacht en de Dokter (The Patient, Their Complaint and The Doctor). Inaugural Lecture. University of Groningen, Groningen, The Netherlands. Retrieved from http://www.rug.nl/corporate/nieuws/archief/archief2011/promoties_oraties/15_OratieBerger on 28 November 2011.

Bourgueil Y., Marek A. \& Mousques J. (2005) The participation of nurses in primary care in six European countries, Ontario and Quebec. Issues in Health Economics (IRDES) 95, 112.

Britt E. \& Blampied N.M. (2010) Motivational interview training: a pilot study of the effects on practitioner and patient behaviour. Behavioural and Cognitive Psychotherapy 38, 239-244.

Britt E., Hudson S.M. \& Blampied N.M. (2004) Motivational interviewing in health settings: a review. Patient Education and Counseling 53, 147-155.

Coleman T. (2000) Using video-recorded consultations for research in primary care: advantages and limitations. Family Practice 17, 422-427.

Davey B. \& Robinson S. (2002) Taking a degree after qualifying as a registered nurse: constraints and effects. Nurse Education Today 22, 624-631.

van Dulmen A.M. (2012) Kom Communiceren (Start Communicating). Inaugural Lecture. Radboud University Nijmegen, Nijmegen, The Netherlands.

van Eijk-Hustings Y.J.L., Daemen L., Schaper N.C. \& Vrijhoef H.J.M. (2011) Implementation of motivational interviewing in a diabetes care management initiative in the Netherlands. Patient Education and Counseling 84, 10-15.

Fairman J.A., Rowe J.W., Hassmiller S. \& Shalala D.E. (2011) Broadening the scope of nursing practice. The New England Journal of Medicine 364, 193-196.

Fukkink R.G., Trienekens N. \& Kramer L.J.C. (2011) Video feedback in education and training: putting learning in the picture. Educational Psychology Review 23, 45-63.

Gardner G., Chang A.M., Duffield C. \& Doubrovsky A. (2013) Delineating the practice profile of advanced practice nursing: a cross-sectional survey using the modified strong model of advanced practice. Journal of Advanced Nursing 69, 1931-1942.

Goldstein H., Rabash J., Plewis I., Draper D., Brown W.J., Yang M., Woodhouse G. \& Healy M. (1998) A User's Guide to MLwiN. Multilevel Models Project, Institute of Education, London.

Heiligers P.J.M., Noordman J., Korevaar J.C., Dorsman S., Hingstman L., van Dulmen A.M. \& de Bakker D.H. (2012) Praktijkondersteuners in de Huisartspraktijk (POH's), Klaar Voor de Toekomst? [Practice Nurses in General Practice, Ready for the Future?]. NIVEL, Utrecht.

Heinrich E. (2011) Diabetes Self-Management: Strategies to Support Patients and Health Care Professionals. PhD thesis, Maastricht University, Maastricht.

Heinrich E., Candel M.J.J.M., Schaper N.C. \& de Vries N.K. (2010) Effect evaluation of a motivational interviewing based counselling strategy in diabetes care. Diabetes Research and Clinical Practice 90, 270-278.

Ivers N., Jamtvedt G., Flottorp S., Young J.M., Odgaard-Jensen J., French S.D., O'Brien M.A., Johansen M., Grimshaw J. \& Oxman A.D. (2012) Audit and feedback: effects on professional practice and patient outcomes. Cochrane Database of Systematic Review 7, $1-227$.

Katon W., von Korff M., Lin E. \& Simon G. (2001) Rethinking practitioner roles in chronic illness: the specialist, primary care physician and the practice nurse. General Hospital Psychiatry 23, 138-144.

Kemper K.J., Foy J.M., Wissow L. \& Shore S. (2008) Enhancing communication skills for pediatric visits through on-line training using video demonstrations. BMC Medical Education 8, 1-8.

Knight K.M., McGowan L., Dickens C. \& Bundy C. (2006) A systematic review of motivational interviewing in physical health care settings. British Journal of Health Psychology 11, 319332.

Kurtz S., Silverman J. \& Draper J. (2005) Teaching and Learning Communication Skills in Medicine. Radcliffe Publishing, Oxford, San Francisco. 
Noordman, J., Weijden, T. van der, Dulmen, S. van. Effects of video-feedback on the communication, clinical competence and motivational interviewing skills of practice nurses:
pre-test posttest control group study. Journal of Advanced Nursing: 2014, 70(10), 2272-2283

Lane C. (2002) The Behaviour Change Counselling Index (BECCI). Manual for Coding Behaviour Change Counselling. Retrieved from http://motivationalinterview.net/library/BECCIManual.pdf on 18 January 2012.

Lane C., Huws-Thomas M., Hood K., Rollnick S., Edwards K. \& Robling M. (2005) Measuring adaptations of motivational interviewing: the development and validation of the behaviour change counselling index (BECCl). Patient Education and Counseling 56, 166173.

Martins R.K. \& McNeil D.W. (2009) Review of motivational interviewing in promoting health behaviors. Clinical Psychology Review 29, 283-293.

Miller W.R. \& Mount K.A. (2001) A small study of training in motivational interviewing: does one workshop change clinician and client behavior? Behavioural and Cognitive Psychotherapy 29, 457-471.

Miller W.R. \& Rollnick S. (2002) Motivational Interviewing: Preparing People for Change, 2nd edn. The Guilford Press, New York.

Noldus L.P., Trienes R.J., Hendriksen A.H., Jansen H. \& Jansen R.G. (2000) The ObserverVideo-Pro: new software for the collection, management and presentation of timestructured data from videotapes and digital media films. Behavior Research Methods, Instruments, \& Computers 32, 197-206.

Noordman J., Verhaak P., van Beljouw I. \& van Dulmen S. (2010) Consulting room computers and their effect on GP-patient communication: comparing two periods of computer use. Family Practice 27, 644-651.

Noordman J., Verhaak P. \& van Dulmen S. (2011) Web-enabled video-feedback: a method to reflect on the communication skills of experienced physicians. Patient Education and Counseling 82, 335-340.

Noordman J., van der Lee I., Nielen M., Vlek H., van der Weijden T. \& van Dulmen S. (2012a) Do trained practice nurses apply motivational interviewing techniques in primary care consultations? Journal of Clinical Medicine Research 4, 393-401.

Noordman J., van der Weijden T. \& van Dulmen S. (2012b) Communication-related behavior change techniques used in face-to-face lifestyle interventions in primary care: a systematic review of the literature. Patient Education and Counseling 89, 227-244.

Noordman J., de Vet E., van der Weijden T. \& van Dulmen S. (2013) Motivational interviewing within the different stages of change: an analysis of practice nurse-patient consultations aimed at promoting a healthier lifestyle. Social Science \& Medicine 87, 6067.

Olde Hartman T.C., Rijswijk E., van Dulmen S., van Weel-Baumgarten E., van Lucassen P.L.B.J. \& van Weel C. (2013) How patients and family physicians communicate about persistent medically unexplained symptoms: a qualitative study of video-recorded consultations. Patient Education and Counseling 90, 354-360.

Ozcakar N., Mevsim V., Guldal D., Gunvar T., Yildirim E., Sisli Z. \& Semin I. (2009) Is the use of videotape recording superior to verbal feedback alone in the teaching of clinical skills? BMC Public Health 9, 1-5.

Pfister-Minogue K.A. \& Salveson C. (2010) Training and experience of public health nurses in using behavior change counseling. Public Health Nursing 27, 544-551.

Rice V.H. \& Stead L.F. (2009) Nursing interventions for smoking cessation. Cochrane Database of Systematic Reviews 1, 1-58.

Richards A., Carley J., Jenkins-Clarke S. \& Richards D.A. (2000) Skill mix between nurses and doctors working in primary-care delegation or allocation: a review of the literature. International Journal of Nursing Studies 37, 185-197.

Richardson A. \& Cunliffe L. (2003) New horizons: the motives, diversity and future of 'nurse led' care. Journal of Nursing Management 11, 80-84.

Roter D.L., Larson S., Shinitzky H., Chernoff R., Serwint J.R., Adamo G. \& Wissow L. (2004) Use of an innovative video feedback technique to enhance communication skills training. Medical Education 38, 145-157.

Rubak S., Sandboek A., Lauritzen T. \& Christensen B. (2005) Motivational interviewing: a systematic review and meta-analysis. British Journal of General Practice 55, 305-312.

Sim J. \& Wright C.C. (2005) The kappa statistic in reliability studies: use, interpretation and sample size requirements. Physical Therapy 85, 257-268. 
Tame S.L. (2013) The effect of continuing professional education on perioperative nurses' relationships with medical staff: findings from a qualitative study. Journal of Advanced Nursing 69, 817-827.

van Thiel J., Ram P. \& van Dalen J. (2000) MAAS-Global Manual. Maastricht University, Maastricht. Retrieved from http://www.hag.unimaas.nl/Maas-Global_2000/GB/MAASGlobal-2000-EN.pdf on 18 January 2012.

Towle A., Godolphin W., Grams G. \& LaMarre A. (2006) Putting informed and shared decision making into practice. Health Expectations 9, 321-332.

Veloski J., Boex J.R., Grasberger M.J., Evans A. \& Wolfson D.B. (2006) Systematic review of the literature on assessment, feedback and physicians' clinical performance: BEME Guide No.7. Medical Teacher 28, 117-128.

Voogdt-Pruis H. (2011) Cardiovascular Prevention. Nurses and Doctors Working Together. PhD thesis, Maastricht University, Maastricht.

van Weel-Baumgarten E., Bolhuis S., Rosenbaum M. \& Silverman J. (2013) Bridging the gap: how is integrating communication skills with medical content throughout the curriculum valued by students? Patient Education and Counseling 90, 177-183. 
Noordman, J., Weijden, T. van der, Dulmen, S. van. Effects of video-feedback on the communication, clinical competence and motivational interviewing skills of practice nurses: pre-test posttest control group study. Journal of Advanced Nursing: 2014, 70(10), 2272-2283

\section{TABLES AND FIGURES}

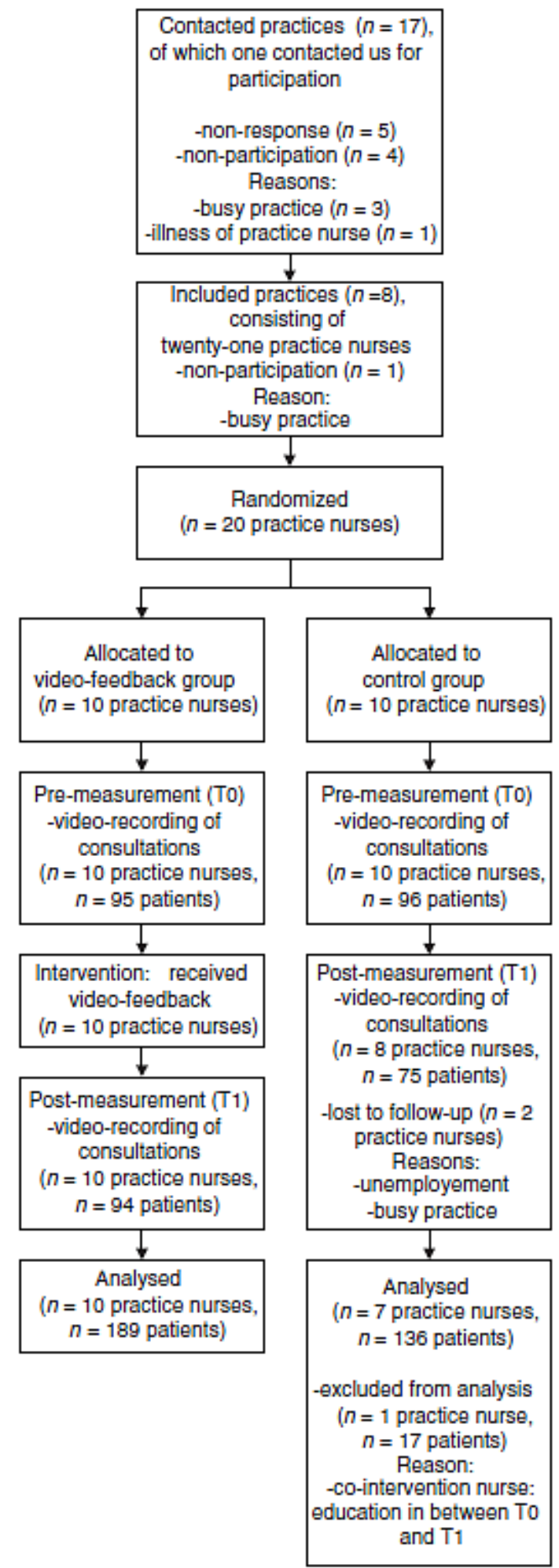

Figure 1 Flow chart of the inclusion procedure. 
Noordman, J., Weijden, T. van der, Dulmen, S. van. Effects of video-feedback on the communication, clinical competence and motivational interviewing skills of practice nurses: a pre-test posttest control group study. Journal of Advanced Nursing: 2014, 70(10), 2272-2283

Table 1 Characteristics of practice nurses $(n-17)$, patients $(n-163)$ and nurses' generic communication, clinical competence and motivational interviewing skills at pre-measurement (T0), in control and video-feedback group ( $n-163)$.

\begin{tabular}{|c|c|c|c|}
\hline Characteristics & $\begin{array}{l}\text { Video- } \\
\text { feedback } \\
\text { group }\end{array}$ & $\begin{array}{l}\text { Control } \\
\text { group }\end{array}$ & $\begin{array}{l}P \\
\text { value }\end{array}$ \\
\hline Practice nurses $(n)$ & 10 & 7 & - \\
\hline Female $(\%)$ & 100 & 100 & - \\
\hline Age in years, mean (sD) & $40(5.9)$ & $46(5 \cdot 3)$ & 0.06 \\
\hline $\begin{array}{l}\text { Experience in years, } \\
\text { mean (sD) }\end{array}$ & $4 \cdot 2(2 \cdot 4)$ & $5 \cdot 6(3.3)$ & 0.34 \\
\hline $\begin{array}{l}\text { Prior posteducational } \\
\text { training } \\
\text { in motivational } \\
\text { interviewing (\%) }\end{array}$ & 100 & 43 & $<0.01^{*}$ \\
\hline Patients $(n)$ & 95 & 68 & - \\
\hline Female $(\%)$ & 52 & 49 & 0.70 \\
\hline Age in years, mean (sD) & $65(11.6)$ & $59(14 \cdot 1)$ & $<0.01^{*}$ \\
\hline \multicolumn{4}{|l|}{ Ethnicity $(\%)$} \\
\hline Dutch & 87 & 69 & $<0.01^{*}$ \\
\hline Western immigrants & 8 & 7 & \\
\hline Non-Western immigrants & 4 & 21 & \\
\hline 'Missing' & 1 & 3 & \\
\hline $\begin{array}{l}\text { Consultation duration } \\
\text { (minutes) }\end{array}$ & $20 \cdot 59$ & $23-42$ & 0.06 \\
\hline $\begin{array}{l}\text { Type of consultation } \\
\text { ( } \% \text { first consultations) }\end{array}$ & $10 \%$ & $16 \%$ & $0 \cdot 18$ \\
\hline \multicolumn{4}{|c|}{$\begin{array}{l}\text { Practice nurses' generic comm unication skills (MAAS-global), mean } \\
\text { scores (sD) }\end{array}$} \\
\hline Introduction & $1.4(1.11)$ & $1.6(1.25)$ & 0.31 \\
\hline Follow-up consultation & $3 \cdot 6(1.21)$ & $4.0(1.38)$ & 0.13 \\
\hline Request for belp & $0.7(1.20)$ & $1.0(1.35)$ & 0.41 \\
\hline Physical examination & $3.9(1.29)$ & $4.1(1.58)$ & 0.13 \\
\hline Diagnosis & $3.1(1.25)$ & $3.5(1.06)$ & $0-46$ \\
\hline Management & $3.7(1.11)$ & $3.6(1.01)$ & $0 \cdot 16$ \\
\hline $\begin{array}{l}\text { Evaluation of } \\
\text { consultation }\end{array}$ & $1.3(1.45)$ & $1 \cdot 1(1.26)$ & 0.58 \\
\hline Exploration & $4.3(0.91)$ & $4.7(0.70)$ & 0.13 \\
\hline Emotions & $1.6(1.60)$ & $1.5(1.61)$ & 0.55 \\
\hline Information giving & $3.7(0.90)$ & $4 \cdot 0(0 \cdot 74)$ & 0.17 \\
\hline Summarizations & $2.4(1.65)$ & $2.0(1.69)$ & 0.20 \\
\hline Structuring & $4.2(0.85)$ & $4.4(0.96)$ & $0.04^{*}$ \\
\hline Empathy & $5 \cdot 1(0.41)$ & $5.2(0.52)$ & 0.22 \\
\hline
\end{tabular}

Practice nurses' clinical competence (MAAS-global), mean scores

(s)

$\begin{array}{llll}\text { History taking } & 4.1(1.26) & 4.3(1.14) & 0.45 \\ \text { Physical examination } & 4.7(0.62) & 4.7(0.59) & 0.92 \\ \text { Diagnosis } & 4.8(0.65) & 4.7(0.58) & 0.34 \\ \text { Management } & 4.7(0.72) & 4.7(0.60) & 0.13\end{array}$

Practice nurses' motivational interviewing \$kills (BECCI), mean scores (s)

Domain
1. Agenda setting and
$2 \cdot 1(0 \cdot 63)$
$2.3(0.55)$ permission seeking 
Noordman, J., Weijden, T. van der, Dulmen, S. van. Effects of video-feedback on the communication, clinical competence and motivational interviewing skills of practice nurses: a

Table 1 (Continued).

\begin{tabular}{llll}
\hline & $\begin{array}{l}\text { Video- } \\
\text { feedback } \\
\text { group }\end{array}$ & $\begin{array}{l}\text { Control } \\
\text { group }\end{array}$ & $\begin{array}{l}P \\
\text { value }\end{array}$ \\
\hline $\begin{array}{c}\text { Characteristics } \\
\begin{array}{c}\text { The why and how of } \\
\text { change in behaviour }\end{array}\end{array}$ & $1.6(0.64)$ & $1.6(0.73)$ & 0.32 \\
$\begin{array}{c}\text { 3. The whole } \\
\text { consultation }\end{array}$ & $2.1(0.74)$ & $2.1(0.80)$ & 0.70 \\
$\begin{array}{c}\text { 4. Talk about targets } \\
\text { Mean sum score }\end{array}$ & $1.8(0.83)$ & $2 \cdot 0(0.80)$ & 0.51 \\
\hline
\end{tabular}

"Significant difference between video-feedback group and control group $(P<0.05)$; an independent $t$-test for continuous variables (e.g. age) and a chi-square test for dichotomous and categorical variables (e.g pror posteducational training in motivational interviewing ethnicity) were used.

'Ethnicity' according to CBS definition (http:/Www.cbs.nl).

1-20 cases of the MAAS-global were excluded due to missing data (i.e. not applicable items of the MAAS-global were coded as missing). Scale of the MAAS-global: 0 - not present, 1 - poor, 2 - unsatisfactory, 3 - doubtful, 4 - satisfactory, 5 - good, 6 - excellent. Scale of the BECCI: 0 - not at all, 1 - minimally, 2 - to some extent, 3 - a good deal, 4 - agreat extent.

BECCI, Behaviour Change Counselling Index.

Table 2 Regression coefficients (model 1) of practice nurses' generic communication skills (according to the MAAS-global) at pre-measurement (T0) vs. postmeasurement (T1) in control and video-feedback group, corrected for patient characteristics.

\begin{tabular}{|c|c|c|c|c|c|}
\hline $\begin{array}{l}\text { Generic communication skills } \\
\text { (MAAS-global) }\end{array}$ & $\begin{array}{l}\text { Regression coefficient } \\
(95 \% \mathrm{CI}) \\
\text { Pre- (T0) vs. } \\
\text { postmeasurement (T1) }\end{array}$ & $\begin{array}{l}\text { Regression coefficient } \\
(95 \% \mathrm{CI}) \\
\text { Control vs. } \\
\text { video-feedback group }\end{array}$ & $\begin{array}{l}\text { Regression coefficient } \\
(95 \% \mathrm{CI}) \\
\text { Interaction term between } \\
\text { measurement and group }\end{array}$ & $P$ value & $\mathrm{ICC}$ \\
\hline Introduction ( $n=312)$ & $0.20(-0.82$ to 1.01$)$ & $0.02(-1.02$ to 1.04$)$ & $-0.05(-1.23$ to 1.17$)$ & 0.93 & 0.06 \\
\hline Follow-up consultation $(n=270)$ & $-0.24(-0.54$ to 0.42$)$ & $-0.23(-0.76$ to 0.60$)$ & $0.39(-0.50$ to 0.75$)$ & $0 \cdot 22$ & $0 \cdot 18$ \\
\hline Request for help $(n=307)$ & $0.05(-0.69$ to 0.73$)$ & $-0.33(-0.79$ to 0.56$)$ & $1.66(-0.15$ to 1.77$)$ & $<0.01 \%$ & 0.00 \\
\hline Physical examination $(n=290)$ & $-0.57(-0.53$ to 0.29$)$ & $-0.14(-0.85$ to 0.73$)$ & $0.86(-0.30$ to 0.77$)$ & $<0 \cdot 01^{*}$ & $0 \cdot 28$ \\
\hline Diagnosis $(n=311)$ & $-0.62(-0.50$ to 0.26$)$ & $-0.31(-0.58$ to 0.42$)$ & $0.39(-0.40$ to 0.60$)$ & 0.13 & $0 \cdot 10$ \\
\hline Management $(n=312)$ & $-0.44(-0.44$ to 0.28$)$ & $0.04(-0.48$ to 0.49$)$ & $0.04(-0.46$ to 0.48$)$ & 0.88 & $0 \cdot 14$ \\
\hline Evaluation of consultation $(n=311)$ & $-0.73(-1.09$ to 0.50$)$ & $0.16(-0.89$ to 1.04$)$ & $0.84(-0.59$ to 1.48$)$ & $0 \cdot 16$ & 0.09 \\
\hline Exploration $(n=314)$ & $-0.66(-0.52$ to 0.26$)$ & $-0.29(-0.55$ to 0.41$)$ & $0.48(-0.39$ to 0.64$)$ & 0.07 & $0 \cdot 12$ \\
\hline Emotions ( $n=313$ ) & $-0.13(-0.78$ to 0.68$)$ & $0.15(-0.96$ to 1.12$)$ & $-0.15(-1.04$ to 0.89$)$ & 0.78 & $0 \cdot 16$ \\
\hline Information giving $(n=314$ ) & $-0.52(-0.48$ to 0.28$)$ & $-0.26(-0.42$ to 0.32$)$ & $0.61(-0.34$ to 0.64$)$ & $0.02 *$ & 0.02 \\
\hline Summarizations ( $n=314$ ) & $0.24(-0.78$ to 1.10$)$ & $0.33(-0.50$ to 0.64$)$ & $0.02(-0.74$ to 0.76$)$ & 0.96 & $0 \cdot 23$ \\
\hline Structuring $(n=312)$ & $-0.11(-0.37$ to 0.33$)$ & $-0.17(-0.51$ to 0.43$)$ & $0.35(-0.38$ to 0.54$)$ & $0 \cdot 14$ & $0 \cdot 18$ \\
\hline Empathy $(n=312)$ & $-0.35(-0.22$ to 0.15$)$ & $-0.03(-0.21$ to 0.21$)$ & $0.15(-0.22$ to 0.26$)$ & 0.22 & 0.08 \\
\hline
\end{tabular}

"Significant difference between experimental and control group, at postmeasurement (T1) compared with pre-measurement (T0) $(P<0.05)$, using multilevel linear regression analysis (except for the items 'Introduction', 'Request for help', 'Evaluation of consultation' and 'Emotions' for which multilevel logistic regression analysis was used).

11-55 cases were excluded due to missing data (i.e. not applicable items were coded as missing).

ICC, intraclass correlation on practice nurse level; CI, confidence interval. 
Noordman, J., Weijden, T. van der, Dulmen, S. van. Effects of video-feedback on the communication, clinical competence and motivational interviewing skills of practice nurses: a pre-test posttest control group study. Journal of Advanced Nursing: 2014, 70(10), 2272-2283

nivel

Table 3 Regression coefficients (model 1) of practice nurses' clinical competence (according to the MAAS-global) at pre-measurement (T0) vs. postmeasurement (T1) in control and video-feedback group, corrected for patient characteristics.

\begin{tabular}{|c|c|c|c|c|c|}
\hline $\begin{array}{l}\text { Clinical competence } \\
\text { (MAAS-global) }\end{array}$ & $\begin{array}{l}\text { Regression coefficient } \\
(95 \% \mathrm{CI}) \\
\text { Pre- (T0) vs. } \\
\text { postmeasurement (T1) }\end{array}$ & $\begin{array}{l}\text { Regression coefficient } \\
(95 \% \mathrm{CI}) \\
\text { Control vs. } \\
\text { video-feedback group }\end{array}$ & $\begin{array}{l}\text { Regression coefficient }(95 \% \mathrm{CI}) \\
\text { Interaction term between } \\
\text { measurement and group }\end{array}$ & $P$ value & ICC \\
\hline History taking ( $n=313$ ) & $-0.16(-0.40$ to 0.34$)$ & $-0.13(-0.53$ to 0.46$)$ & $0.29(-0.42$ to 0.56$)$ & $0 \cdot 25$ & $0 \cdot 10$ \\
\hline Physical examination $(n=291)$ & $0.02(-0.23$ to 0.24$)$ & $0.03(-0.31$ to 0.32$)$ & $0.13(-0.28$ to 0.32$)$ & 0.40 & $0 \cdot 18$ \\
\hline Diagnosis $(n=313)$ & $0.11(-0.18$ to 0.20 & $0.05(-0.20$ to 0.21$)$ & $-0.01(-0.25$ to 0.24$)$ & 0.93 & 0.05 \\
\hline Management $(n=312)$ & $0.01(-0.25$ to 0.26$)$ & $-0.06(-0.27$ to 0.25$)$ & $0.10(-0.32$ to 0.35$)$ & 0.54 & 0.04 \\
\hline
\end{tabular}

12-34 cases were excluded due to missing data (i.e. not applicable items were coded as missing). Results are based on multilevel linear regression analysis.

ICC, intraclass correlation on practice nurse level; CI, confidence interval.

Table 4 Regression coefficients (model 1) of practice nurses' motivational interviewing skills (according to the BECCI) at pre-measurement (T0) vs. postmeasurement (T1) in control and video-feedback group, corrected for patient characteristics.

\begin{tabular}{|c|c|c|c|c|c|}
\hline $\begin{array}{l}\text { Motivational interviewing } \\
\text { skills (BECCI) }\end{array}$ & $\begin{array}{l}\text { Regression coefficient } \\
(95 \% \mathrm{CI}) \\
\text { Pre- (T0) vs. } \\
\text { postmeasurement (T1) }\end{array}$ & $\begin{array}{l}\text { Regression coefficient } \\
(95 \% \mathrm{CI}) \\
\text { Control vs. } \\
\text { video-feedback group }\end{array}$ & $\begin{array}{l}\text { Regression coefficient }(95 \% \mathrm{CI}) \\
\text { Interaction term between } \\
\text { measurement and group }\end{array}$ & $P$ value & $\mathrm{ICC}$ \\
\hline \multicolumn{6}{|l|}{ Domains } \\
\hline $\begin{array}{l}\text { 1. Agenda setting and } \\
\text { permission seeking }\end{array}$ & $-0.32(-0.26$ to 0.19$)$ & $-0.22(-0.27$ to 0.21$)$ & $0.39(-0.24$ to 0.35$)$ & $0.01^{*}$ & 0.04 \\
\hline $\begin{array}{l}\text { 2. The why and how of } \\
\text { change in behaviour }\end{array}$ & $-0.25(-0.28$ to 0.22$)$ & $0.04(-0.35$ to 0.37$)$ & $0.17(-0.30$ to 0.35$)$ & 0.32 & $0 \cdot 16$ \\
\hline 3. The whole consultation & $-0.49(-0.43$ to 0.26$)$ & $0.04(-0.32$ to 0.33$)$ & $0.01(-0.45$ to 0.45$)$ & 0.96 & 0.00 \\
\hline 4. Talk about targets & $-0.42(-0.27$ to 0.27$)$ & $-0.08(-0.33$ to 0.31$)$ & $0.13(-0.41$ to 0.47$)$ & 0.57 & 0.00 \\
\hline BECCI mean sum score & $-0.37(-0.33$ to 0.23$)$ & $-0.06(-0.29$ to 0.27$)$ & $0.18(-0.33$ to 0.40$)$ & $0 \cdot 34$ & 0.03 \\
\hline
\end{tabular}

"Significant difference between video-feedback and control group, at postmeasurement (T1) compared with pre-measurement (T0) $(P<0.05)$, using multilevel linear regression analysis.

11 cases were excluded due to missing data on patient characteristics ( $\mathrm{n}=314$ for all BECCI domains).

BECCI, Behaviour Change Counselling Index; ICC, intraclass correlation on practice nurse level; CI, confidence interval. 